\title{
Temperature Dependence of Dielectric Constant and Loss Tangent in Methyl Ammonium Aluminium Alum
}

\author{
ARVIND KUMAR RAWAT, AANCHAL RAWAT and \\ TRILOK CHANDRA UPADHYAY
}

Physics Department, H.N.B. Garhwal University, Srinagar (Garhwal) U.K-246174, India

arvindsgfi@gmail.com

Received 4 January 2016 / Accepted 20 January 2016

\begin{abstract}
The ferroelectric phase transition of MASD alum was studied by model of pseudospinlattice coupled mode which has been modified by including cubic and quartic phonon anharmonic interaction terms. With the help of double-time temperature dependent Green's function method and modified Hamiltonian, expressions for dielectric constant, soft mode frequency and loss tangent has been evaluated. By fitting model values of physical quantities in the theoretical expressions, temperature dependence of dielectrc, soft mode frequency and loss tangent have been obtained and compared with literature values which shows a good agreement.
\end{abstract}

Keywords: Green's function, Dielectric constant, Anharmonic interactions, Loss tangent

\section{Introduction}

It is well known that ferroelectric materials find potential applications in technology due to their peculiar properties. They are used in memory devices as like infrared detectors, optical modulators, large capacitors etc ${ }^{1}$. Methyl ammonium aluminum alum (MASD), $\left(\mathrm{CH}_{3} \mathrm{NH}_{3}\right) \mathrm{Al}\left(\mathrm{SO}_{4}\right) \cdot 12 \mathrm{H}_{2} \mathrm{O}$ is ferroelectric below $177 \mathrm{~K}$. In high temperature phase the $\mathrm{CH}_{3} \mathrm{NH}_{3}$ groups are found to be symmetrically oriented so that they are indistinguishable from each other ${ }^{2}$. But in low-temperature phase $\mathrm{CH}_{3} \mathrm{NH}_{3}$ groups are separately arranged which look like a dumbbell. In high temperature phase $\mathrm{CH}_{3} \mathrm{NH}_{3}$ groups are oriented in a disordered fashion, as if their average orientation looks spherical, while below $\mathrm{T}_{\mathrm{c}}$ in the ordered state, they take on a particular orientation and become distinguishable and symmetric. The ordering of methyl group in MASD gives rise to order-disorder type of phase transition. Singh et al., ${ }^{3}$ have done synthesis and characterization studies on alum. Derby ${ }^{4}$ has carried out crystal growth studies of methyl ammonium aluminium alum. Ghanem et al., ${ }^{5}$ have studied experimentally the effect of doping different polymers on these alums. Sachdeva et al., ${ }^{6}$ have done crystal growth studies on methyl ammonium aluminium alum. Basra et al., ${ }^{7}$ have done electrical conductivity in methyl ammonium 
aluminium alum. Sajan et al., ${ }^{8}$ have studied physical properties of polymer mixed alums. Previously Chaudhury et al., ${ }^{9}$ have studied ferroelectric transition in methyl ammonium aluminium alum using pseudospin-lattice coupled mode model with fourth order phonon anharmonic interaction term. They have not considered third-order phonon anharmonic interaction term. They have decoupled correlations at an early stage so that some important interactions disappeared from their results.

The present work include pseudospin-lattice coupled mode model along with third and fourth-order phonon anharmonic interactions terms. We shall decouple the correlations at proper stage. In MASD alum, since there is no isotope effect hence the term $B_{i j}$ (which has been considered by earlier research ${ }^{2}$ ) makes almost no contribution to the system and so we have not included this term in our model of MASD (as suggested by Chunlei et al., ${ }^{10}$ also). By using double-time thermal Green's function method along with modified model Hamiltonian, expressions for renormalized soft mode frequency, dielectric constant and loss tangent, shift and width have been obtained. By fitting model values of various quantities in derived expressions, their thermal variations have been calculated. The theoretically calculated expressions for soft mode frequency have been compared with correlated values of soft mode frequency obtained from experimental results of dielectric constant for MASD crystal reported by Pepinsky et $a l^{12}$.

Theoretical results for loss tangent and dielectric constant for MASD crystal have been compared with experimentally reported results of Pepinsky et $a l^{12}$.

\section{Model Hamiltonian}

In the case of methyl ammonium aluminium alum, it is concluded from neutron diffraction experiment that the asymmetric distribution of the $\mathrm{H}$ bonds around the sulphate groups might be a cause of the ferroelectric transition. Concentrating our attention on the proton subsystem associated with the active ions, the simplest form of modified model Hamiltonian is expressed as;

$$
\begin{aligned}
& H=-2 \Omega \sum_{i} S_{i}^{x}-\frac{1}{2} \sum_{i j} J_{i j} S_{i}^{Z} S_{j}^{Z}+\frac{1}{4} \sum_{k} \omega_{k}\left(A_{k} A_{k}^{+}+B_{k} B_{k}^{+}\right)-\sum_{i k} V_{i k} S_{i}^{z} A_{k} \\
& +\sum_{k_{1} k_{2} k_{3}} V^{4}\left(k_{1}, k_{2}, k_{3}\right) A_{k_{1}} A_{k_{2}} A_{k_{3}}+\sum_{k_{1} k_{2} k_{3} k_{4}} V^{4}\left(k_{1}, k_{2}, k_{3}, k_{4}\right) A_{k_{1}} A_{k_{2}} A_{k_{3}} A_{k_{4}} .
\end{aligned}
$$

In the above equation, last two terms have been added by us. $S_{i}^{\alpha}(\alpha=x, y, z)$ is the $\alpha^{\text {th }}$ component of the pseudospin $\overrightarrow{\mathrm{S}}_{\mathrm{I}}, \Omega$ is proton tunneling frequency. $\mathrm{J}_{\mathrm{ij}}$ is spin -spin interaction constant. $A_{k}$ and $B_{k}$ are position and momentum operators, $\omega_{k}$ is harmonic phonon frequency, $\mathrm{V}^{(3)}$ and $\mathrm{V}^{(4)}$ are third- and fourth order atomic force constants.

\section{Green's Function, Shift and Width}

Following Zubarev ${ }^{13}$, we consider the Green's function

$$
G_{i j}\left(t-t^{\prime}\right)=\left\langle\left\langle S_{i}^{z}(t) ; S_{j}^{z}\left(t^{\prime}\right)\right\rangle\right\rangle,=-i \theta\left(t-t^{\prime}\right)\left\langle\left[S_{i}^{z}(t) ; S_{j}^{z}\left(t^{\prime}\right)\right]\right\rangle
$$

Differentiating Green's function Eq. (2) two times with respect to times t and t respectively, Fourier transforming and writing in Dyson's equation form is obtained. 


$$
\begin{gathered}
G_{i j}(\omega)=\frac{\Omega\left\langle S_{i}^{x}\right\rangle \delta_{i j}}{\pi\left[\omega^{2}-\tilde{\Omega}^{2}-P(\omega)\right]} \text {, where } \\
\tilde{\Omega}^{2}=4 \Omega^{2}+\frac{i\left\langle[F(t)], S_{j}^{y}\left(t^{\prime}\right)\right\rangle}{\left\langle S_{j}^{x}\right\rangle}, \text { and } \\
P(\omega)=\frac{\pi}{\Omega\left\langle S_{i}^{x}\right\rangle}\left\langle\left\langle F(t) ; F^{\prime}\left(t^{\prime}\right)\right\rangle\right\rangle \text {, with } \\
\left\langle\left\langle F_{i}(t) ; F_{j}^{\prime}\left(t^{\prime}\right)\right\rangle\right\rangle={ }^{2} J_{i j}^{2}\left\{\left\langle\left\langle S_{i}^{x} S_{j}^{z} ; S_{i}^{x} S_{j}^{z}\right\rangle\right\rangle+\left\langle\left\langle S_{i}^{x} S_{j}^{z} ; S_{i}^{z} S_{j}^{x}\right\rangle\right\rangle+\left\langle\left\langle S_{i}^{z} S_{j}^{x} ; S_{i}^{x} S_{j}^{z}\right\rangle\right\rangle+\left\langle\left\langle S_{i}^{z} S_{j}^{x} ; S_{i}^{z} S_{j}^{x}\right\rangle\right\rangle\right\} \\
+\Omega^{2} V_{i k}^{2}\left\{\left\langle\left\langle A_{k} S_{i}^{x} ; S_{i}^{x} A_{i}^{+}\right\rangle\right\rangle\right\} .
\end{gathered}
$$

Above equation consist higher order Green's functions which are evaluated after decoupling them by using decoupling scheme $\langle A B ; C D\rangle=\langle A B\rangle\langle C D\rangle+\langle A C\rangle\langle B D\rangle+\langle A D\langle B C\rangle\rangle$. Simpler Green's functions are evaluated using zeroth-order approximation: i.e. neglecting higher order Green's functions in them. In this way $\left\langle\left\langle F(t) ; F\left(t^{\prime}\right)\right\rangle\right\rangle$ is evaluated. Its real part is called shift $\Delta(\omega)$ and imaginary part is called width $\Gamma(\omega)$. Hence Eq. (3) becomes

$$
G_{i j}(\omega)=\frac{\Omega\left\langle S_{i}^{x}\right\rangle \delta_{i j}}{\pi\left[\omega^{2}-\tilde{\Omega}^{2}-2 i \Omega \Gamma(\omega)\right]} .
$$

where, $\quad \hat{\Omega}^{2}=\tilde{\Omega}^{2}+\Delta(\omega)$,

$$
\begin{gathered}
\tilde{\Omega}^{2}=a^{2}+b^{2}-b c, \\
a=J\left\langle S_{i}^{z}\right\rangle, \\
b=2 \Omega, \\
c=J\left\langle S^{x}\right\rangle,
\end{gathered}
$$

shift $\Delta(\omega)$ and width $\Gamma(\omega)$ are obtained as

$$
\Delta(\omega)=\frac{a^{4}}{2 \Omega\left(\omega^{2}-\widetilde{\Omega}^{2}\right)}+\frac{b^{2} c^{2}}{2 \Omega\left(\omega^{2}-\widetilde{\Omega}^{2}\right)}+\frac{V_{i k}^{2} N_{K} a^{2}}{2 \Omega\left(\omega^{2}-\widetilde{\Omega}^{2}\right)}+\frac{2 V_{i k}^{2}\left\langle S_{1 i}^{x}\right\rangle \omega_{k} \delta_{k-k^{\prime}}\left(\omega^{2}-\tilde{\widetilde{\omega}}_{k}^{2}\right)}{\left(\omega^{2}-\tilde{\widetilde{\omega}}_{k}^{2}\right)+4 \omega_{k}^{2} \Gamma_{k}^{2}(\omega)},
$$

and

$$
\Gamma(\omega)=\frac{\pi a^{4}}{4 \Omega \tilde{\Omega}}[\delta(\omega-\tilde{\Omega})-\delta(\omega+\tilde{\Omega})]+\frac{b^{2} c^{2}}{4 \Omega \tilde{\Omega}}[\delta(\omega-\tilde{\Omega})-\delta(\omega+\tilde{\Omega})]+\frac{2 V_{i k}^{2}\left\langle S_{1 i}^{x}\right\rangle \omega_{k} \delta_{k-k^{\prime}}\left(\omega^{2}-\tilde{\widetilde{\omega}}_{k}^{2}\right)}{\left(\omega^{2}-\tilde{\tilde{\omega}}_{k}^{2}\right)+4 \omega_{k}^{2} \Gamma_{k}^{2}(\omega)} .
$$

In Eqs. (13) and (14) $\tilde{\widetilde{\omega}}_{k}$ and $\Gamma_{k}(\omega)$ are modified phonon frequency and phonon width respectively. These appear in the evaluation of phonon Green's function $\left\langle\left\langle A_{k} ; A_{k}^{+}\right\rangle\right\rangle$which is obtained as 


$$
\left\langle\left\langle A_{k} ; A_{k}^{+}\right\rangle\right\rangle=\frac{\omega_{k} \delta_{k_{k}}}{\omega^{2}-\widetilde{\widetilde{\omega}}_{k}-2 i \omega_{k} \Gamma_{k}(\omega)}
$$

In Eq. (15) $\tilde{\widetilde{\omega}}_{k}$ is obtained as

$$
\tilde{\tilde{\omega}}^{2}=\tilde{\omega}_{k}^{2}+2 \omega_{k} \Delta_{k}(\omega) \text {. }
$$

$\Gamma_{k}(\omega)$ is obtained as

$$
\begin{aligned}
& \Gamma_{k}(\omega)=9 \pi \sum_{k_{1} k_{2}}\left|V^{(3)}\left(k_{1}, k_{2},-k\right)\right|^{2} \frac{\omega_{k_{1}} \omega_{k_{2}}}{\tilde{\omega}_{k_{1}} \tilde{\omega}_{k_{2}}}\left\{\left(n_{k_{2}}+n_{k_{1}}\right) \mid \delta\left(\omega+\tilde{\omega}_{k_{1}}+\tilde{\omega}_{k_{2}}\right)-\delta\left(\omega+\tilde{\omega}_{k_{1}}-\tilde{\omega}_{k_{1}}\right)\right. \\
& \left.\left.\left.+\left(n_{k_{2}}-n_{k_{1}}\right) \times \delta\left(\omega+\tilde{\omega}_{k_{1}}+\tilde{\omega}_{k_{1}}\right)-\delta\left(\omega+\tilde{\omega}_{k_{1}}+\tilde{\omega}_{k_{1}}\right)\right\}\left(\omega+\tilde{\omega}_{k_{1}}+\tilde{\omega}_{k_{1}}\right)\right\}\right] \\
& +48 \pi \sum\left|V\left(k_{1}, k_{2}, k_{3},-k_{4}\right)\right|^{2}\left\{1+n_{k_{1}} n_{k_{2}}+n_{k_{2}} n_{k_{3}}+n_{k_{3}} n_{k_{4}}\right\} \\
& \left.\mid \delta\left(\tilde{\omega}+\tilde{\omega}_{k_{1}}+\tilde{\omega}_{k_{2}}+\tilde{\omega}_{k_{3}}\right)-\delta\left(\tilde{\omega}-\tilde{\omega}_{k_{1}}-\tilde{\omega}_{k_{2}}-\tilde{\omega}_{k_{3}}\right)\right]
\end{aligned}
$$

In Eq. (16) $\Delta_{k}(\omega)$ appears which is obtained as

$$
\begin{aligned}
& \Delta_{k}(\omega)=18 P \sum_{k_{1} k_{2}}\left|V^{3}\left(k_{1} k_{2-k}\right)\right|^{2} \frac{\omega_{k_{1}} \omega_{k_{2}}}{\tilde{\omega}_{k 1} \tilde{\omega}_{k 2}}\left\{\left(n_{k_{1}}+n_{k_{2}}\right) \frac{\tilde{\omega}_{k_{1}}+\tilde{\omega}_{k_{2}}}{\omega^{2}-\left(\tilde{\omega}_{k_{2}}+\tilde{\omega}_{k_{2}}\right)^{2}}+\left(n_{k_{2}}+n_{k_{1}}\right) \frac{\tilde{\omega}_{k_{1}}+\tilde{\omega}_{k_{2}}}{\omega^{2}-\left(\tilde{\omega}_{k_{1}}+\tilde{\omega}_{k_{2}}\right)^{2}}\right\}+48 \mathrm{P} \\
& \sum_{k_{1} k} \mid V^{(4)}\left(k_{1} k_{2} k_{3,-k}\right)^{2} \frac{\omega_{k_{1}} \omega_{k_{2}} \omega_{k_{3}}}{\tilde{\omega}_{k_{1}} \tilde{\omega}_{k_{2}} \tilde{\omega}_{k_{3}}}\left\{\left(1+n_{k_{1}} n_{k_{2}}+n_{k_{2}} n_{k_{3}}+n_{k_{3}} n_{k_{1}}\right) \frac{\tilde{\omega}_{k_{1}}+\tilde{\omega}_{k_{2}}+\tilde{\omega}_{k 3}}{\omega^{2}-\left(\tilde{\omega}_{k_{1}}+\tilde{\omega}_{k_{2}}+\tilde{\omega}_{k_{3}}\right)^{2}}\right. \\
& +3\left(1-n_{k_{2}} n_{k_{1}}+n_{k_{2}} n_{k_{3}}-n_{k_{3}} n_{k_{1}}\right) \frac{\tilde{\omega}_{k_{1}}+\tilde{\omega}_{k_{2}}+\tilde{\omega}_{k_{3}}}{\omega^{2}-\left(\tilde{\omega}_{k_{1}}+\tilde{\omega}_{k_{2}}+\tilde{\omega}_{k_{3}}\right)^{2}}+\text { higher terms. }
\end{aligned}
$$

In Eq. (18) P stands for principal part.

\section{Modified soft mode frequency}

According to Cochran the ferroelectric transition in certain crystals results from freezing of normal mode frequency at transition temperature. The expression for this frequency can be easily obtained from Eq. (8). Putting value of $\Delta(\omega)$ in Eq. (8) and simplifying Eq. (19) obtained as follows.

$$
\hat{\Omega}^{2}=\frac{1}{2}\left[\left(\tilde{\Omega}^{2}+\tilde{\omega}_{k}^{2}\right) \pm\left\{\left(\tilde{\omega}_{k}{ }^{2}-\tilde{\Omega}^{2}\right)^{2}+8 V_{i k}^{2}\left\langle S_{i}^{x}\right\rangle \Omega\right\}^{\frac{1}{2}}\right] .
$$

The transition temperature $\mathrm{T}_{\mathrm{C}}$ can be evaluated by putting the condition that phase transition occurs when $\mathrm{T}$ approaches $\mathrm{T}_{\mathrm{C}}$, i.e. when $\tilde{\Omega}$ becomes zero. From Eq. (9) we get $\sqrt{a^{2}+b^{2}-b c}=0, \mathrm{a}=\mathrm{J}\left\langle S^{Z}\right\rangle$ and $\left\langle S^{Z}\right\rangle=0$ at $\mathrm{T}_{\mathrm{C}}$ so $\mathrm{b}^{2}-\mathrm{bc}=0$, where $\mathrm{c}=J^{\prime}\left\langle S^{X}\right\rangle$ and $\left\langle S^{X}\right\rangle=\tanh \left(\frac{\Omega}{2 k_{B} T_{C}}\right)$ at $\mathrm{T}_{\mathrm{C}}$. Hence we obtain $\tanh \left(\frac{\Omega}{2 k_{B} T_{C}}\right)=\frac{4 \Omega}{\mathrm{J}^{\prime}}$ which gives transition temperature $\mathrm{T}_{\mathrm{C}}$ as 


$$
T_{c}=\frac{\Omega}{2 k_{B} \tanh ^{-1}\left(\frac{4 \Omega}{J^{\prime}}\right)},
$$

Where

$$
J^{\prime}=J+\frac{2 V_{i k}^{2} \omega_{k}^{2}}{\tilde{\omega}_{k}^{2}} .
$$

Above equations show that modified soft mode frequency $\hat{\Omega}_{-}$is function of tunneling frequency $\Omega$ as well as modified phonon anharmonic frequency $\widetilde{\widetilde{\omega}}_{\mathrm{k}}$. The transition temperature $\mathrm{T}_{\mathrm{c}}$ is function of tunneling frequency $\Omega$, spin-lattice interaction constant $\mathrm{V}_{\mathrm{ik}}$ as well as modified phonon frequency $\widetilde{\widetilde{\omega}}_{\mathrm{k}}$. The phonon frequency depends upon phonon anharmonic interactions terms.

\section{Dielectric constant and loss tangent}

The dielectric constant $\in$ is related to susceptibility

$$
\chi \text { as } \in=1+4 \pi \chi \text {. }
$$

In ferroelectric crystals, always, $\in \gg>1$ therefore $\epsilon=4 \pi \chi$

Hence one obtains using Eqs. (24), (22) and Green's function (7) the expression for dielectric constant $\in$ as

$$
\epsilon=-\frac{8 \pi N \mu^{2} \Omega\left\langle S_{i}^{X}\right\rangle}{\left[\left(\omega^{2}-\hat{\Omega}^{2}-2 \Omega i \Gamma(\omega)\right)\right]}
$$

The ferroelectric and dielectric material loses energy in the form of heat, called dielectric tangent loss. This is ratio of real and imaginary parts of dielectric constant. From Eq. (25) we get the following:

$$
\tan \delta=-\frac{2 \Omega \Gamma(\omega)}{\left(\omega^{2}-\hat{\Omega}^{2}\right)}
$$

From Eqs. (25) and (26) we observes that dielectric constant and loss tangent depend on modified soft mode frequency. Hence these depend upon tunneling frequency as well as anharmonic interactions terms.

\section{Numerical calculation and Discussion}

Numerical calculations have been carried out with the help of model values of the physical quantities appearing in expressions, derived for methyl ammonium aluminium alum, i.e. $\Omega=0.7, T_{C}=177 \mathrm{~K}, J^{\prime}=488.99 \mathrm{~cm}^{-1}, V_{i k}=65.56 \mathrm{~cm}^{-1}, N=0.512 \times 10^{21}, \mu \times 10^{18} \mathrm{cgs}=3.513$, $\omega_{k}=5 \mathrm{~cm}^{-1}, C=540 \mathrm{~K}, J=366.75 \mathrm{~cm}^{-1}, A_{k} \times 10^{17}\left(\operatorname{ergK}^{-1}\right)=37.66$. Their temperature variations of shift, width, soft mode frequency, dielectric constant and loss tangent have been calculated. The theoretical variation of soft mode frequency with temperature are compared with values obtained by correlating the experimental data on dielectric constant measurements reported by Pepinsky et al., ${ }^{11}$ for MASD crystal. The variations of dielectric constant and loss tangent have been compared with experimental results of others ${ }^{13}$. Our results agree with experimental results of these researchers [Figures (1,2 and 3)]. 


\section{Conclusion}

Our study shows that the pseudospin-lattice coupled mode model along with third-and fourth-order phonon anharmonic interaction terms explains well the temperature dependences of ferroelectric properties of methyl ammonium aluminium alum. The soft mode frequency is renormalized by phonon anharmonic interaction terms. The modified soft mode frequency clearly explains the nature of phase transition at $177 \mathrm{~K}$ in MASD alum, which agrees with experimental results of Pepinsky et $a l^{12}$.

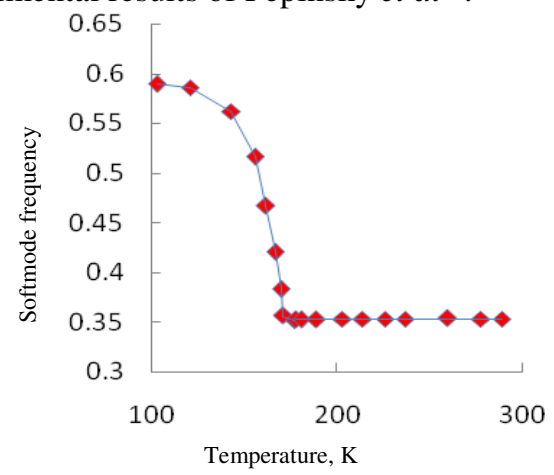

Figure 1. Temperature dependence of soft mode frequency $\hat{\Omega}\left(\mathrm{cm}^{-1}\right)$ of MASD alum (Our results; $\gg$ experimentally correlated results of Pepensky et al ${ }^{12}$ )

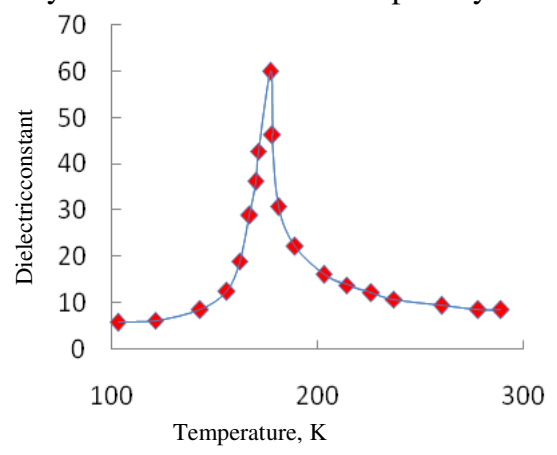

Figure 2. Temperature dependence of dielectric constant $\epsilon$ of MASD alum (- Our results; - Experimental results of Pepensky et $a l^{12}$ )

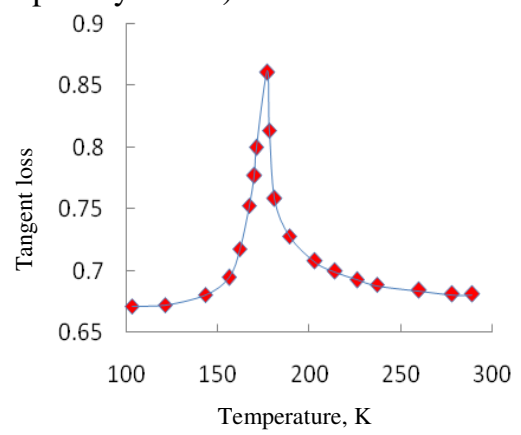

Figure 3. Temperature dependence of tangent loss $(\delta)$ of MASD alum (- Our results; - Experimental results of Pepensky et $a l^{12}$ ) 


\section{Acknowledgement}

Authors are grateful to Eminent Physicist Prof. B S Semwal ( Former HOD) for his valuable suggestions and to Prof. S C Bhatt (HOD), Prof. N S Negi (H P Univ., Shimla), Prof. Vinay Gupta (Delhi Univ.), Prof. K K Verma (A U, Faizabad), Prof R P Gairola, Prof. U C Naithani, Prof. G C Joshi and Dr Manish Uniyal for their kind encouragements, One of authors (AKR) is thankful to Dr K P Semwal who taught him computer programme (numerical calculations).

\section{References}

1. Phatak C, Petford- Lang A K, Beleggia M and Graef M De, Phys Rev B, 2013, 214112; DOI:10.1103/PhysRevB.89.214112

2. Chaudhury K R, Nath D, Banerjee S and Chaudhuri B K, Phys Rev B, 1982, 26, 6276; DOI:10.1103/PhysRevB.26.6276

3. Singh P K, Bhattacharya B, Sachdeva A and Raja Singh, Mater Technol., 2013, 47, 467-471.

4. Derby J J, "Crystal Growth: growth, bulk (Theory and Model)" in: Encyclopedia of Condenced Matter Physics, Eds, Bassani F, J Liedl and Wider P, Acad Press, 2005, 274-282.

5. Ghanem H M E, Saq'uan S, Saadi M and Abdul Jawad S, J Mod Physics, 2011, 2, 1553; DOI:10.4236/jmp.2011.212189

6. Suchdeva A, Singh R, Singh P K and Bhattacharya B, Mater Technol., 2013, (47)4, 467.

7. Basra S M A, Iqbal Z, Rahman K, Rahman H and Ejaz M F, J Appl Res Technol., 2014, 12, 560-567.

8. Sajan S G, Ayesh A S, Zihilif A, Martuscelli E and Zagosta R, Polymer Testing, 2004, 23(7), 739-745; DOI:10.1016/j.polymertesting.2004.04.008

9. Chaudhury K R, Nath D, Banerjee S, and Chaudhuri B K, Phys Rev B, 1982, 25, 627; DOI:10.1103/PhysRevB.25.627

10. Chunlei W, Zikai Q and Jingbo Z, Ferroelectrics, 1988, 77(1), 21-29; DOI:10.1080/00150198808223223

11. Zubarev D N, Sov Phys Usp., 1960, 3, 320-345; DOI:0038-5670/3/3/R02

12. Pepinsky R, Jona F and Shirane G, Phys Rev B, 1956, 102, 1181. 